\title{
About asymmetric noisy chaotic maps
}

\author{
Salah H Abid *, Hasan M. Hasan \\ Mathematics Department, Education College, Al-Mustansiriya University, Baghdad, Iraq \\ *Corresponding author E-mail: abidsalah@gmail.com
}

Copyright $\odot 2014$ Salah H Abid, Hasan M. Hasan. This is an open access article distributed under the Creative Commons Attribution License, which permits unrestricted use, distribution, and reproduction in any medium, provided the original work is properly cited.

\begin{abstract}
In this paper we will introduce three families of asymmetric maps, and discuss some dynamical properties for these families in the deterministic case, and noisy case. New mixed noisy chaotic map will be suggested and then studied with some details.
\end{abstract}

Keywords: Asymmetric Maps, Lyapunov Exponent Mixed Noisy Chaotic Map, Random Dynamical System, And Random Fixed Point.

\section{Basic concepts}

Let $(\Omega, \mathcal{F}, \mathbb{P})$ denoted a probability space , and let $\left(\vartheta^{t}\right)_{t \in \mathbb{Z}^{+}}: \Omega \rightarrow \Omega$ be an invertible map such that $\vartheta^{t}$ and $\vartheta^{-t}$ are measurable $, \vartheta \mathbb{P}=\mathbb{P}, \vartheta^{0}=i d_{\Omega}, \vartheta^{n+m}=\vartheta^{n} o \vartheta^{m}$, and all $B \in \mathcal{F}$ with $\vartheta^{-1}(B)=B$ then $\mathbb{P}(B) \in\{0,1\}$, we will define the ergodic process $\xi_{n}(\omega)=\xi\left(\vartheta^{n}(\omega)\right), n \in \mathbb{Z}^{+}$, where $\xi: \Omega \rightarrow \mathbb{R}$ is measurable function.

Definition 1.1: (random dynamical system - discrete time) [1].

Let $(\Omega, \mathcal{F}, \mathbb{P})$ denoted a probability space then the random dynamical system is define as a random difference equation $x_{t+1}(\omega)=\varphi\left(t, x_{0}, \xi_{0}(\omega)\right)=\varphi\left(1, x_{t}, \xi_{t}(\omega)\right)$, where $t \in \mathbb{Z}^{+} \varphi: \mathbb{Z}^{+} \times \mathbb{R} \times \Omega \rightarrow \mathbb{R}$ and $\xi_{t}(\omega)$ defined above. Such that i) $\quad \varphi\left(0, x_{0}, \xi_{0}(\omega)\right)=x_{0}(\omega)$

ii) $\quad \varphi\left(n+m, x_{0}, \xi_{0}(\omega)\right)=\varphi\left(n, x_{m}, \xi_{m}(\omega)\right) o \varphi\left(m, x_{0}, \xi_{0}(\omega)\right)$

Definition 1.2: (random fixed point) [6].

A random fixed point $p(\omega)$ of a random dynamical system $\varphi$ on $\mathbb{R}$ is a random variable $p(\omega): \Omega \rightarrow \mathbb{R}$ such that. Almost surely (a.s.) $\quad p(\omega)=\varphi\left(1, p(\omega), \xi_{0}(\omega)\right)$

The random fixed point $p(\omega)$ is attracting with probability $P_{r}(p(\omega)$ is attracting $)=P$ if $P_{r}\left(\lim _{t \rightarrow \infty} \mid x_{t}(\omega)-\right.$ $p(\omega) \mid=0)=P$, for all $x_{0}(\omega) \in(p(\omega)-\varepsilon, p(\omega)+\varepsilon)$ for same $\varepsilon>0$

If $P=1$ (i.e. $\lim _{t \rightarrow \infty}\left|x_{t}(\omega)-p(\omega)\right|=0$ a.s. ) then $p(\omega)$ is called globally attracting [6].

Lemma 1.1: let $p(\omega)$ be a random fixed point to the random dynamical system $\varphi$ on $\mathbb{R}$, and $\varphi$ partial differentiable at $p(\omega)$ then

$P_{r}(p(\omega)$ is attracting $)=P_{r}\left(\left|\frac{\partial \varphi\left(t, x, \xi_{t}(\omega)\right)}{\partial p(\omega)}\right|<1\right)$

\section{Proof:}

Let $\left|\frac{\partial \varphi\left(t, x, \xi_{t}(\omega)\right)}{\partial p(\omega)}\right|<1$ then $\left|\frac{x_{1}(\omega)-p(\omega)}{x_{0}(\omega)-p(\omega)}\right| \leq A$ for same $0<A<1$ and same $\varepsilon>0$. and any $x_{0}(\omega) \in(p(\omega)-\varepsilon$, $p(\omega)+\varepsilon)$ then we have $\left|x_{1}(\omega)-p(\omega)\right| \leq A\left|x_{0}(\omega)-p(\omega)\right|$. by mathematical induction we get $\left|x_{t}(\omega)-p(\omega)\right| \leq$ $A^{t}\left|x_{0}(\omega)-p(\omega)\right| \quad$ and hence $\lim _{t \rightarrow \infty}\left|x_{t}(\omega)-p(\omega)\right|=0 \quad$, which implies to $P_{r}\left(\left|\frac{\partial \varphi\left(t, x, \xi_{t}(\omega)\right)}{\partial p(\omega)}\right|<1\right)=P_{r}\left(\lim _{t \rightarrow \infty}\left|x_{t}(\omega)-p(\omega)\right|=0\right)=P_{r}(p(\omega)$ is attracter $)$. 


\section{Some dynamical properties in deterministic case}

\subsection{The family of asymmetric tent map}

The dynamical system for the asymmetric tent map can be defined as follows:

$x_{t+1}=T_{a, \mu}\left(x_{t}\right)=\left\{\begin{array}{cl}\mu \frac{x_{t}}{a} & \text { if } 0 \leq x_{t} \leq a \\ \mu \frac{1-x_{t}}{1-a} & \text { if } a<x_{t} \leq 1\end{array}\right.$

Where $T_{a, \mu}:[0,1] \rightarrow[0,1]$ and $0 \leq \mu \leq 1,0 \leq a \leq 1$.

Theorem 2.1: For a dynamical system in (1).

1) If $\mu<a$ then the system has one attracting fixed point $p=0$.

2) If $\mu=a$ then all points of the interval $[0, a]$ represent fixed point to the system and all points from the interval $(a, 1]$ represent eventually fixed points and it is orbit have only two points

3) If $a<\mu<1-a$ then the system has two fixed points $p_{1}=0$ replying and $p_{2}=\frac{\mu}{\mu+(1-a)}$ attracting.

4) If $\mu>a$ and $\mu>1-a$ then there are no any attracting fixed or periodic point and the system becomes chaotic, see Lyapunov exponent figure (1).

Proof: Clear.

The satisfaction of the above properties can be seen from the bifurcation diagrams in figure (2)

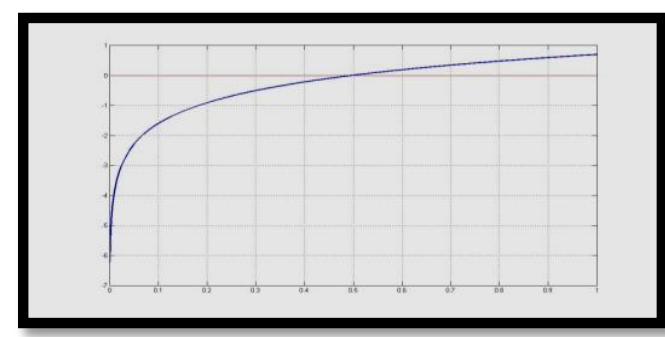

(a)

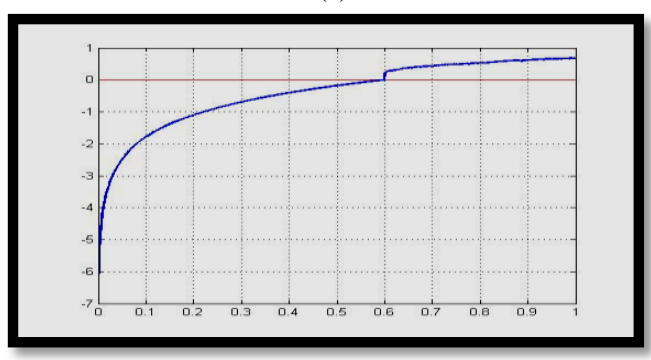

(c)

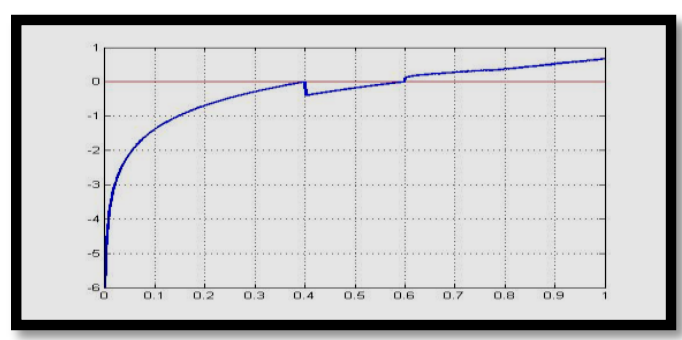

(b)

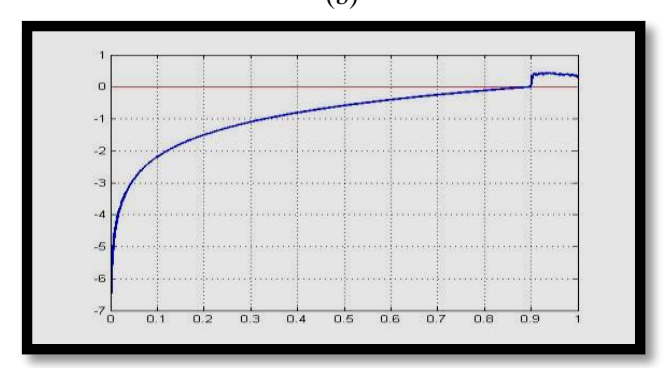

(d)

Fig. 1: Lyapunov exponent of asymmetric tent map, $x_{-}$axes represent the values of $\mu$ and $y_{-}$axes represent the values of Lyapunov exponent , (a) $a=0.5$, (b) $a=0.4$, (c) $a=0.6$, (d) $a=0.9$

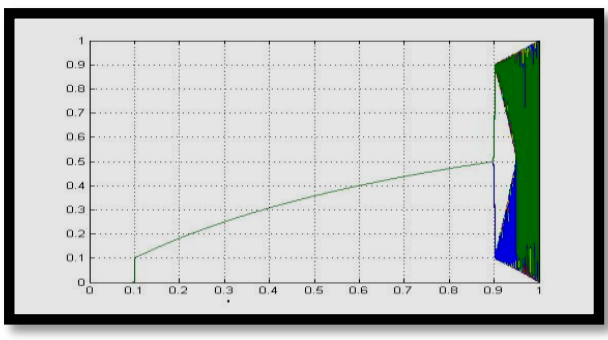

(a)

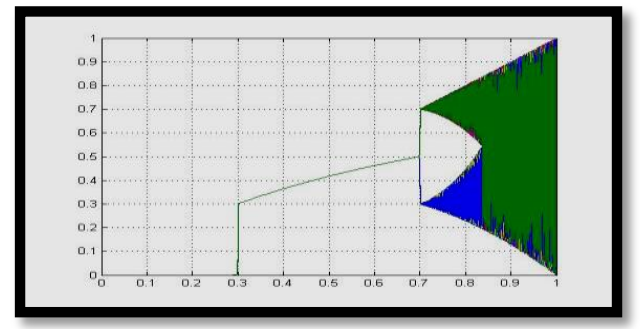

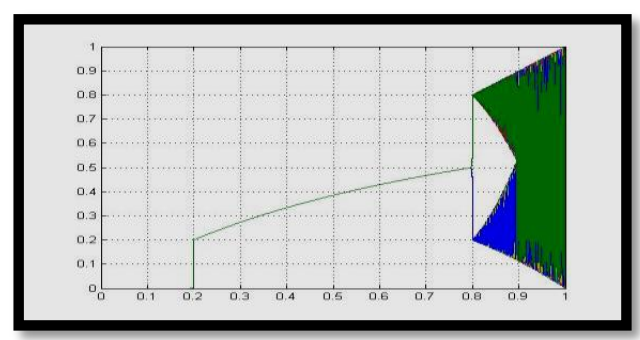

(b)

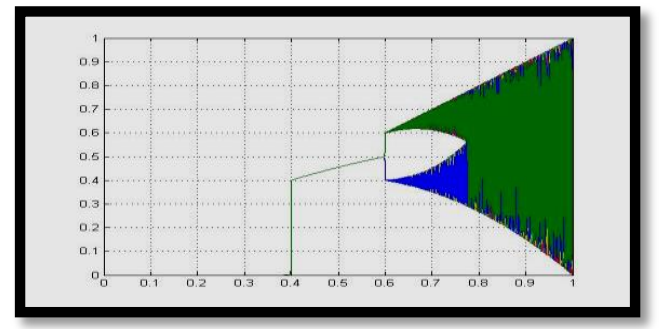


(c)

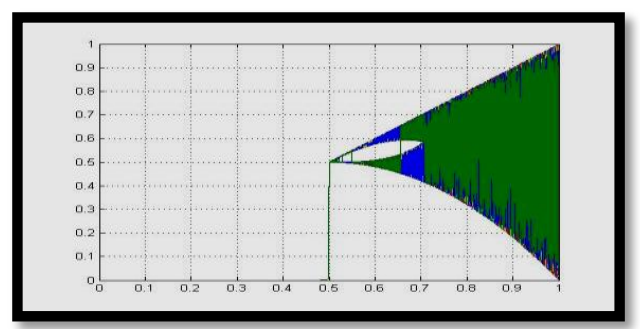

(e)

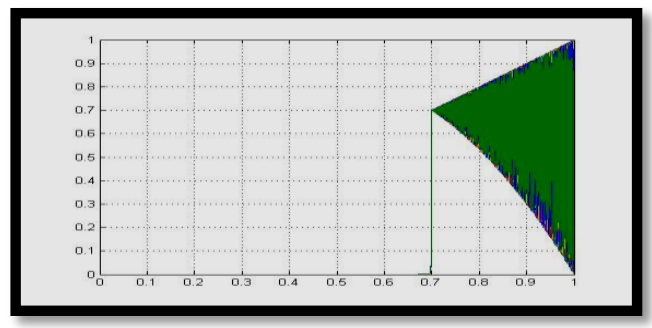

(g) (d)

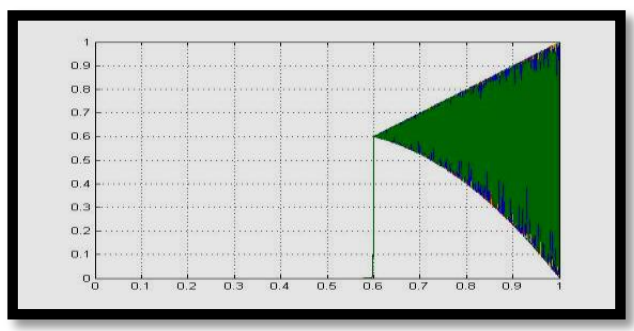

(f)

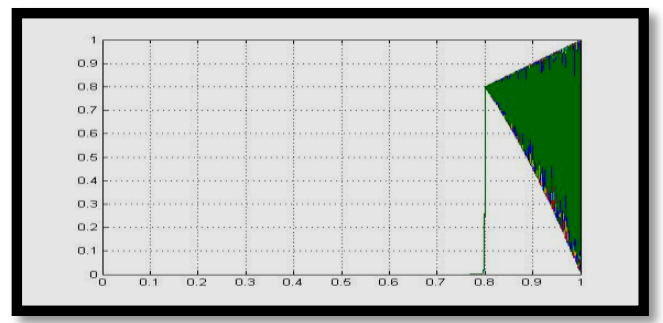

(h)

Fig. 2: bifurcation diagram of asymmetric tent map family, $\mathrm{x}$-axes represent the values of $\mu$ and $\mathrm{y}$-axes represent the values of $\mathrm{x}_{\mathrm{t}+1}$ where $\mathrm{x}_{\mathrm{t}+1} \in[0,1]$ , (a) $a=0.1$, (b) $a=0.2$, (c) $a=0.3$, (d) $a=0.4$, (e) $a=0.5$, (f) $a=0.6$,(g) $a=0.7$, (h) $a=0.8$

\subsection{The family of asymmetric logistic map}

The dynamical system for asymmetric logistic map can be defined as follows:

$x_{t+1}=G_{a, \mu}\left(x_{t}\right)=\left\{\begin{array}{cc}\mu \frac{x_{t}}{2 a}\left(1-\frac{x_{t}}{2 a}\right) & \text { if } 0 \leq x_{t} \leq a \\ \mu\left(\frac{x_{t}+1-2 a}{2-2 a}\right)\left(1-\frac{x_{t}+1-2 a}{2-2 a}\right) & \text { if } a<x_{t} \leq 1\end{array}\right.$

Where $G_{a, \mu}:[0,1] \rightarrow[0,1]$ and $0 \leq a \leq 1,0 \leq \mu \leq 4$.

Theorem 2.2: For a dynamical system in (2)

1) If $\mu<2 a$ then the system has on attracting fixed point $p=0$.

2) If $2 a<\mu \leq 4 a$ then the system has two fixed points $p_{1}=0$ is replying and $p_{2}=\frac{2 a \mu-4 a^{2}}{\mu}$ is attracting and $p_{1}, p_{2} \in[0, a]$.

3) If $2 a<\mu<2 a+\sqrt{4 a^{2}+3(2-2 a)^{2}}$ then the second fixed point

$p_{2}=\frac{\left(\frac{2 a \mu}{(2-2 a)^{2}}-1\right)-\sqrt{\left(\frac{2 a \mu}{(2-2 a)^{2}}-1\right)^{2}+\left(\frac{4\left(\mu^{2}-2 a \mu^{2}\right)}{(2-2 a)^{4}}\right)}}{\frac{2 \mu}{(2-2 a)^{2}}}$ is attracting and $p_{2} \in[0,1]$

Proof: Clear.

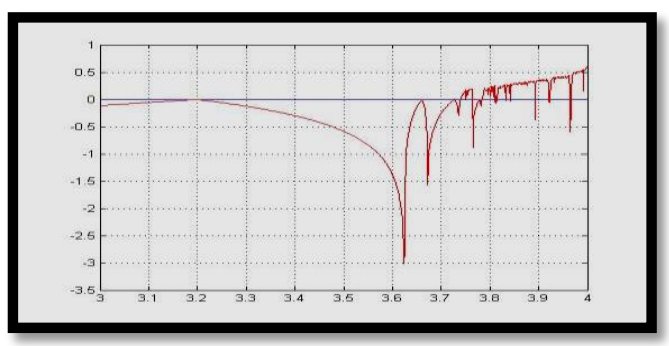

(a)

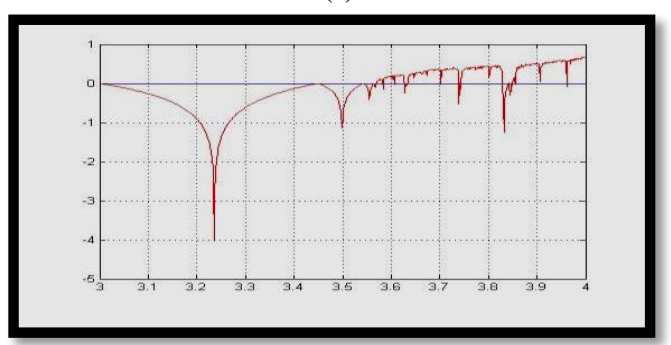

(c)

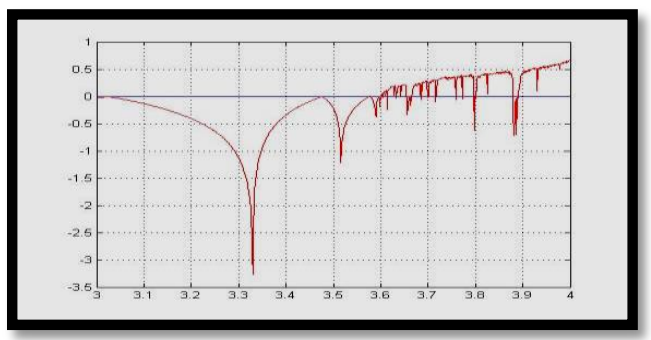

(b)

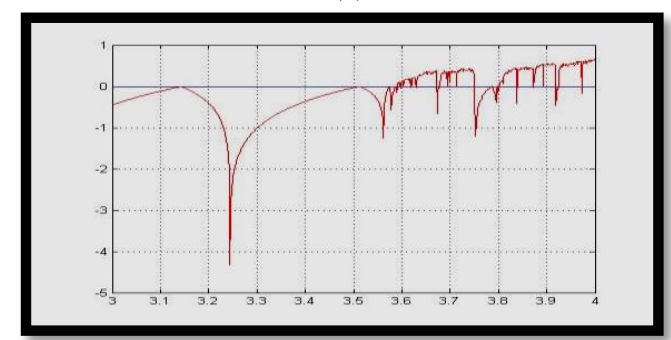

(d)

Fig. 3: Lyapunov exponent of asymmetric logistic map, $x_{-}$axes represent the values of $\mu$ and $y_{-}$axes represent the values of Lyapunov exponent , (a) $a=0.2$, (b) $a=0.4$, (c) $a=0.5$, (d) $a=0.7$. 


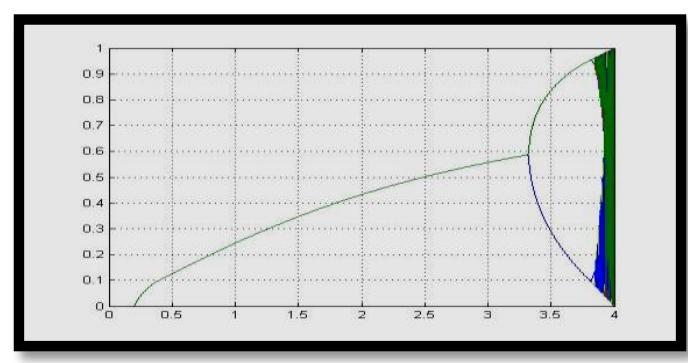

(a)

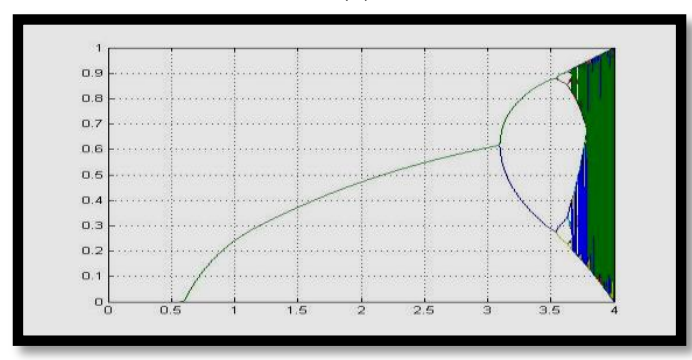

(c)

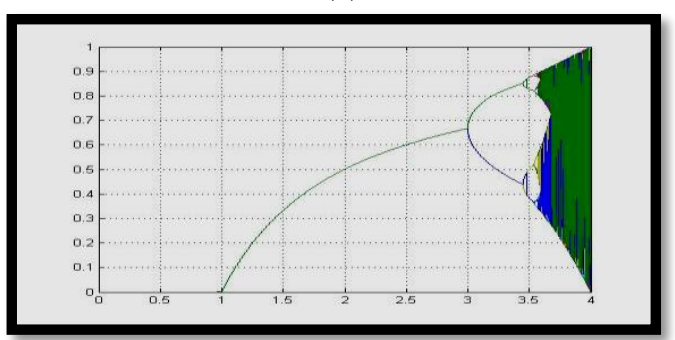

(e)

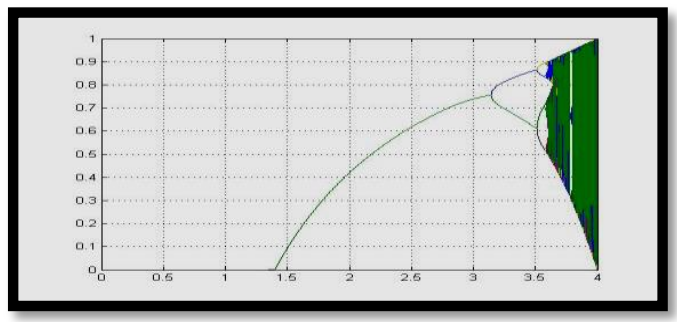

(g)

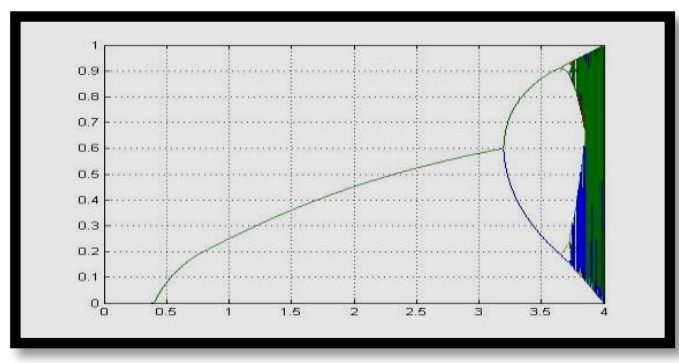

(b)

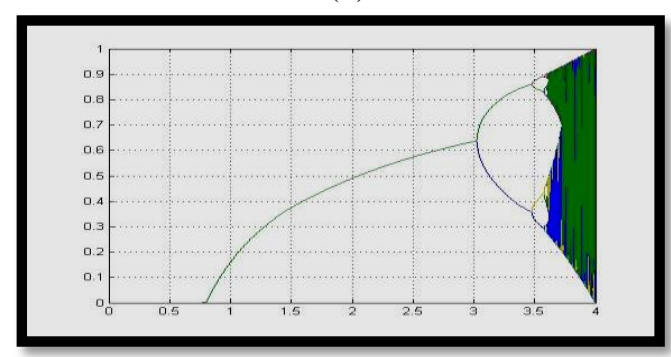

(d)

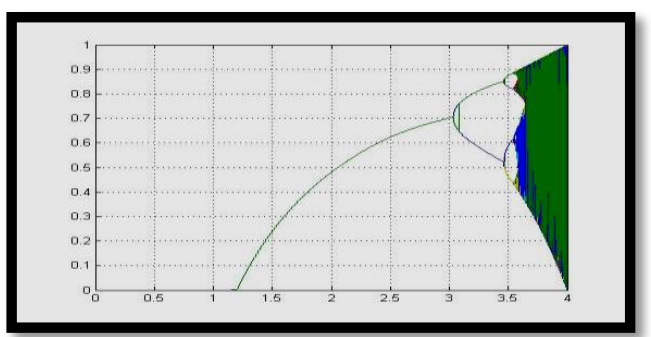

(f)

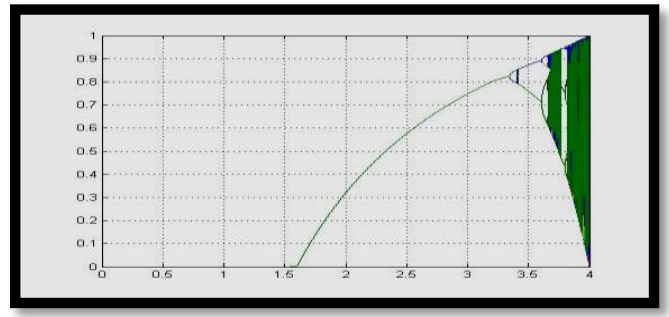

(h)

Fig. 4: bifurcation diagram of asymmetric logistic map family, $\mathrm{x}$-axes represent the values of $\mu$ and $\mathrm{y}$-axes represent the values of $\mathrm{x}_{\mathrm{t}+1}$ with $\mathrm{x}_{\mathrm{t}+1} \in[0,1]$ , (a) $a=0.1$, (b) $a=0.2$, (c) $a=0.3$, (d) $a=0.4$, (e) $a=0.5$, (f) $a=0.6$, (g) $a=0.7$, (h) $a=0.8$.

\subsection{The family of mixed logistic-tent map}

The dynamical system for the mixed logistic-tent map can be defined as follows:

$x_{t+1}=T G_{a, \mu}\left(x_{t}\right)= \begin{cases}\mu \frac{x_{t}}{2 a}\left(1-\frac{x_{t}}{2 a}\right) & \text { if } 0 \leq x_{t} \leq a \\ \mu\left(\frac{1-x_{t}}{4(1-a)}\right) & \text { if } a<x_{t} \leq 1\end{cases}$

Where $T G_{a, \mu}:[0,1] \rightarrow[0,1]$ and $0 \leq a \leq 1,0 \leq \mu \leq 4$.

Theorem 2.3: For a dynamical system in (3)

1) If $\mu<2 a$ then the system has one attracting fixed point $p=0$.

2) If $2 a<\mu<4 a$ then the system has two fixed points $p_{1}=0$ is replying and $p_{2}=\frac{2 a \mu-4 a^{2}}{\mu}$ is attracting, and $p_{1}, p_{2} \in[0, a]$.

3) If $\mu=4 a$ then the second fixed point $p_{2}=a$ is attracting.

4) If $4 a<\mu<4(1-a)$ then $p_{2}=\frac{\mu}{\mu+4(1-a)}$ is attracting, and $p_{2}$ replying if $\mu>\max \{4 a, a(1-a)\}$. 


\section{Proof:}

The proofs of (1), (2) and (4) are clear. To prove (3), $p_{2}=\frac{2 a \mu-4 a^{2}}{\mu}=\frac{8 a^{2}-4 a}{4 a}=a$

To show that $p_{2}$ is attracter we must find open interval $J=\left(p_{2}-\varepsilon, p_{2}+\varepsilon\right), \varepsilon>0$, such that for any $x_{0} \in J$ then the orbit $\left\{x_{t}\right\} \rightarrow p_{2}$, where $\left\{x_{t}\right\}$ the orbit of $x_{0}$.

Claim $: J=(0,1):$ At first let $x_{0} \in(0, a)$ since $G T_{a, 4 a}\left(x_{t}\right) \leq G T_{a, 4 a}(a)=a$, for every $x_{t} \in(0, a)$, then $\left\{x_{t}\right\}$ bounded from above and $a$ represent $\sup \left(\left\{x_{t}\right\}\right)$, and since $G T^{\prime}{ }_{a, 4 a}\left(x_{t}\right)=\frac{4 a}{2 a}\left(1-\frac{x}{a}\right)>0$ for every $x_{t} \in(0, a)$ then $\left\{x_{t}\right\}$ increasing, that implies $\left\{x_{t}\right\}$ converge to $a$. To complete the proof we need to show for any $x_{0} \in(a, 1)$ then $x_{1} \in$ $(0, a)$.

$x_{1}=G T_{a, 4 a}\left(x_{0}\right)=a\left(\frac{1-x_{0}}{1-a}\right)$, since $0<a<x_{0}<1$ then $0<1-x_{0}<1-a<0$, and hence $\frac{1-x_{0}}{1-a}<1$ which implies to $0<x_{1}=a \frac{1-x_{0}}{1-a}<a$.

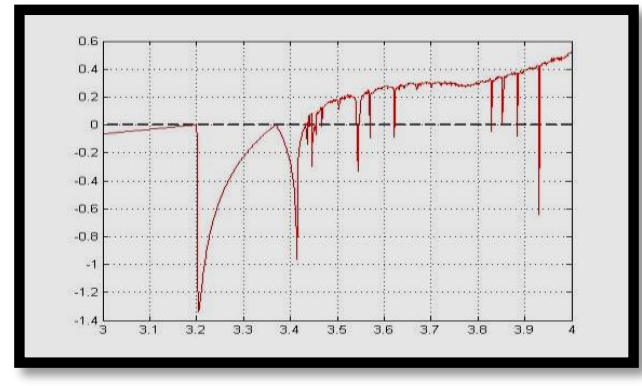

(a)

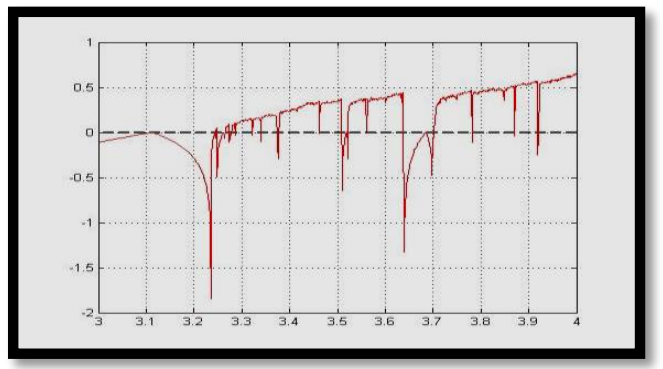

(c)

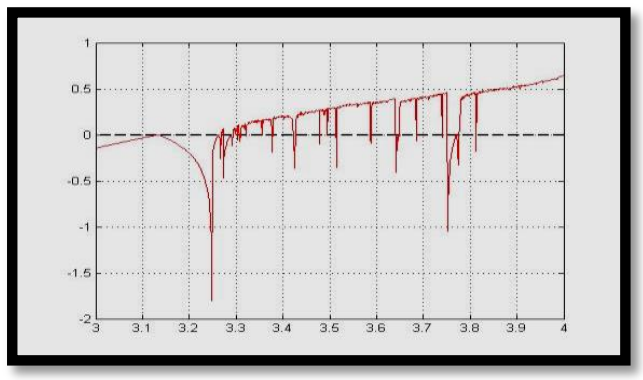

(b)

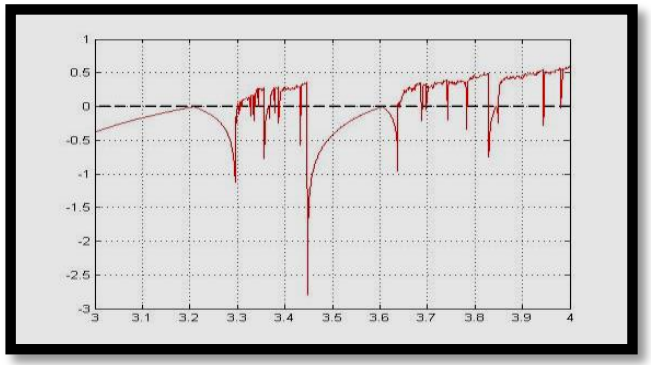

(d)

Fig. 5: Lyapunov exponent of mixed logistic-tent map, $x_{-}$axes represent the values of $\mu$ and $y_{-}$axes form value of Lyapunov exponent , (a) $a=$ 0.2, , (b) $a=0.4$, (c) $a=0.5$, (d) $a=0.7$.

Table 1: Some properties of the asymmetric tent, asymmetric logistic and mixed logistic-tent map

\begin{tabular}{|c|c|c|c|c|c|c|c|c|c|c|c|}
\hline \multirow[t]{2}{*}{ No. } & \multirow[t]{2}{*}{ property } & \multirow[t]{2}{*}{ map } & \multicolumn{9}{|c|}{ value of " $a$ " } \\
\hline & & & 0.1 & 0.2 & 0.3 & 0.4 & 0.5 & 0.6 & 0.7 & 0.8 & 0.9 \\
\hline \multirow{3}{*}{1} & \multirow{3}{*}{$\begin{array}{l}\text { Value of " } \mu \text { " } \\
\text { which Fixed point } \\
\text { becomes unstable }\end{array}$} & A.L & 3.3240 & 3.2000 & 3.0980 & 3.0270 & 3.0000 & 3.0330 & 3.1430 & 3.3430 & 3.6330 \\
\hline & & A.T & 0.9000 & 0.8000 & 0.7000 & 0.6000 & 0.5000 & 0.6000 & 0.7000 & 0.8000 & 0.9000 \\
\hline & & $\begin{array}{l}\text { M.L- } \\
\mathrm{T}\end{array}$ & 3.6000 & 3.2000 & 2.8000 & 2.4000 & 2.0000 & 2.4000 & 2.8000 & 3.2000 & 3.6000 \\
\hline \multirow{3}{*}{2} & \multirow{3}{*}{$\begin{array}{l}\text { Value of " } \mu \text { " } \\
\text { which Chaotic } \\
\text { region begins . }\end{array}$} & A.L & 3.8510 & 3.7400 & & 3.6020 & 3.5700 & 3.5660 & 3.5910 & & \\
\hline & & A.T & 0.9000 & 0.8000 & 0.7000 & 0.6000 & 0.5000 & 0.6000 & 0.7000 & 0.8000 & 0.9000 \\
\hline & & $\begin{array}{l}\text { M. } \\
\text { L-T }\end{array}$ & 3.6560 & 3.4370 & 3.3160 & 3.2680 & 3.2460 & 3.2520 & 3.3010 & 3.4180 & 3.6480 \\
\hline \multirow{3}{*}{3} & \multirow{3}{*}{$\begin{array}{l}\text { Value of " } \mu \text { " } \\
\text { which cycle with } \\
\text { period } 3 \text { appears . }\end{array}$} & A. L & 3.9905 & 3.9645 & 3.9255 & 3.8786 & 3.8285 & 3.7819 & 3.7496 & 3.7490 & 3.8102 \\
\hline & & A. $\mathrm{T}$ & 0.995 & 0.9688 & 0.9280 & 0.8748 & 0.8091 & 0.7294 & 0.7001 & 0.8002 & 0.9004 \\
\hline & & $\begin{array}{l}\text { M. } \\
\text { L-T }\end{array}$ & 3.9860 & 3.9340 & 3.8520 & 3.7520 & 3.6410 & 3.5282 & 3.4489 & 3.4670 & 3.6550 \\
\hline \multirow{3}{*}{4} & \multirow{3}{*}{$\begin{array}{l}\text { Value of " } \mu \text { " } \\
\text { which chaotic } \\
\text { region ends . }\end{array}$} & A. L. & 4 & 4 & 4 & 4 & 4 & 4 & 4 & 4 & 4 \\
\hline & & A. $\mathrm{T}$. & 1 & 1 & 1 & 1 & 1 & 1 & 1 & 1 & 1 \\
\hline & & $\begin{array}{l}\text { M. } \\
\text { L-T }\end{array}$ & 4 & 4 & 4 & 4 & 4 & 4 & 4 & 4 & 4 \\
\hline \multirow{3}{*}{5} & \multirow{3}{*}{$\begin{array}{l}\text { Are there stable } \\
\text { cycles in the } \\
\text { chaotic region? }\end{array}$} & A. L. & Yes & Yes & Yes & Yes & Yes & Yes & Yes & Yes & Yes \\
\hline & & A. T. & No & No & No & No & No & No & No & No & No \\
\hline & & $\begin{array}{l}\text { M. } \\
\text { L-T }\end{array}$ & Yes & Yes & Yes & Yes & Yes & Yes & Yes & Yes & Yes \\
\hline
\end{tabular}




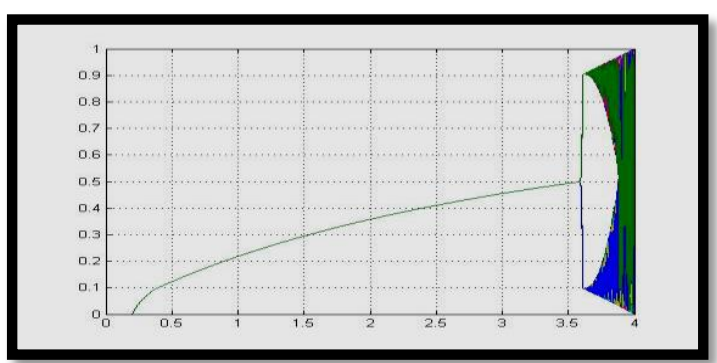

(a)

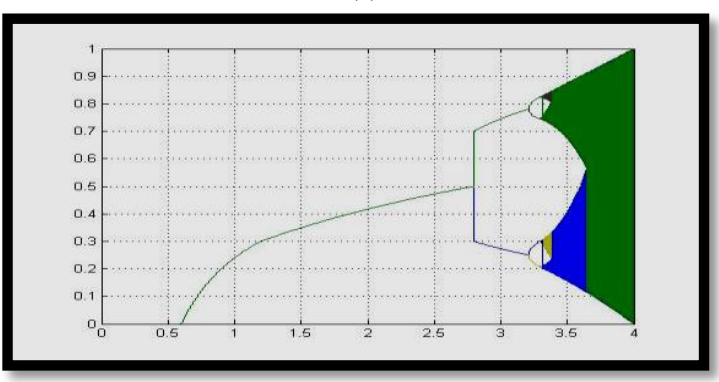

(c)

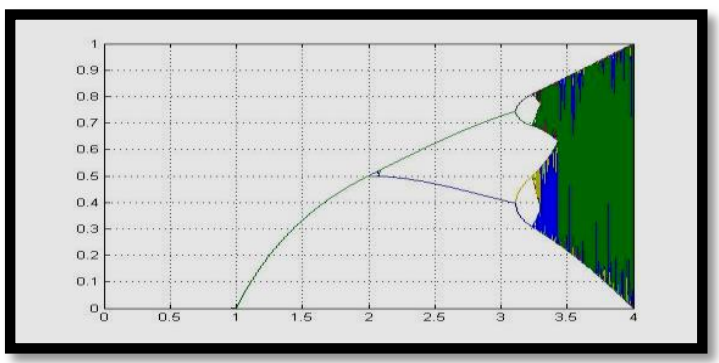

(e)

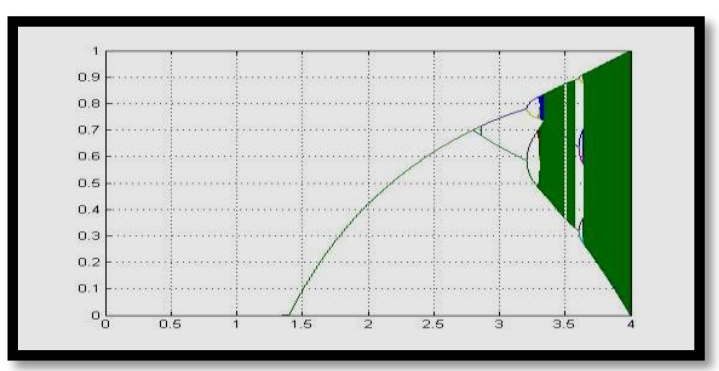

(g)

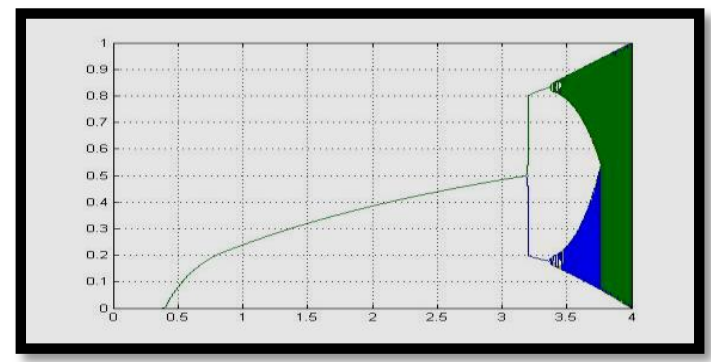

(b)

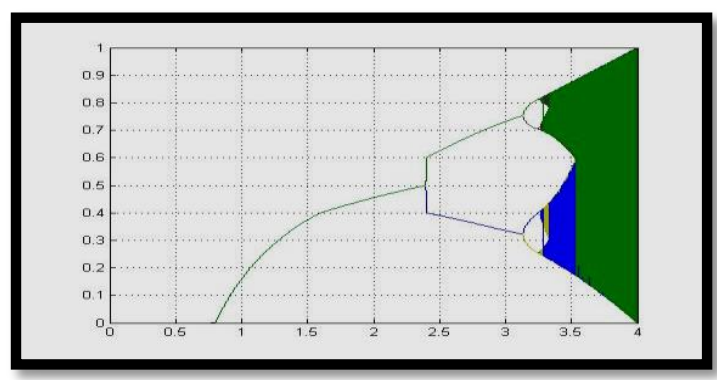

(d)

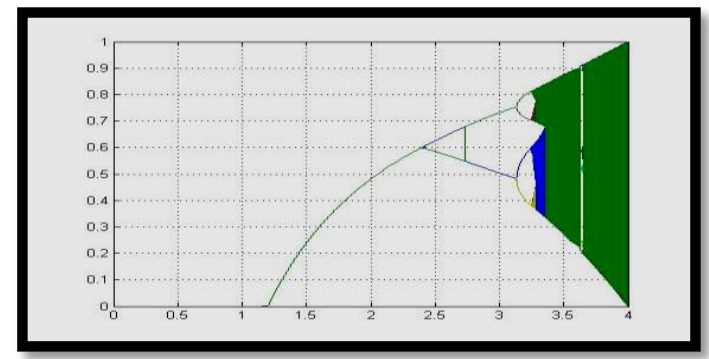

(f)

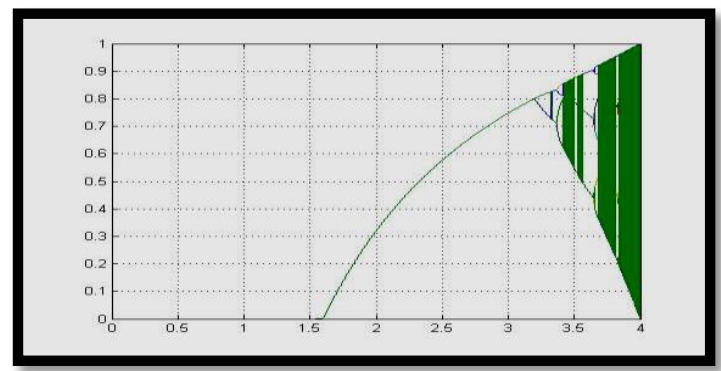

(h)

Fig. 6: bifurcation diagram of mixed logistic-tent map family, $\mathrm{x}$-axes represent the values of $\mu$ and $\mathrm{y}$-axes represent the values of $\mathrm{x}_{\mathrm{t}+1}$ with $\mathrm{x}_{\mathrm{t}+1} \in[0,1]$ , (a) $a=0.1$, (b) $a=0.2$, (c) $a=0.3$,(d) $a=0.4$, (e) $a=0.5$, (f) $a=0.6$,(g) $a=0.7$, (h) $a=0.8$

\section{Some dynamical properties in noisy case}

All maps treated with in previous section were defined on closed interval $[0,1]$, but in the noisy case it is not true generally.

\subsection{The family of noisy asymmetric tent map.}

The noisy dynamical system for asymmetric tent map with additive noise is given by.

$x_{t+1}(\omega)=\tilde{T}_{a, \mu}\left(x_{t}, \xi_{t}(\omega)\right)= \begin{cases}\mu \frac{x_{t}}{a}+\xi_{t}(\omega) & \text { if } 0 \leq x_{t} \leq a \\ \mu \frac{1-x_{t}}{1-a}+\xi_{t}(\omega) & \text { if } a<x_{t} \leq 1\end{cases}$

Where $\tilde{T}_{a, \mu}: \mathbb{R} \times \Omega \rightarrow \mathbb{R},\left\{\xi_{t}(\omega)\right\}$ is a sequence of independent identically distributed (iid) random variables and $0 \leq a \leq 1,0 \leq \mu \leq 1$

Theorem 3.1: For the dynamical system (4), where $\xi_{t}(\omega) \sim \operatorname{Gauss}\left(0, \sigma^{2}\right)$, 
1) If $\mu<a$ then the system has one random fixed point $p(\omega) \sim \operatorname{Gauss}\left(0, \sigma^{2}\right)$, and $P_{r}(p(\omega)$ is attracting) $=$ $P_{r}(\xi(\omega)<a)$, if $\mu>1-a$, and $P_{r}(p(\omega)$ is attracting $)=1$ if $\mu<1-a$

2) If $\mu=a$ then there are infinite number of random fixed points $p(\omega) \sim \operatorname{Gauss}\left(p^{*}, \sigma^{2}\right), p^{*} \in[0, a]$.

$3)$ If $a<\mu<1-a$ then the system has two random fixed points $p_{1}(\omega) \sim \operatorname{Gauss}\left(0, \sigma^{2}\right)$ and $p_{2}(\omega) \sim \operatorname{Gauss}\left(\frac{\mu}{\mu+(1-a)}, \sigma^{2}\right) \quad, \quad$ where $\quad P_{r}\left(p_{1}(\omega)\right.$ is attracting $)=P_{r}(\xi(\omega)>a) \quad$ and $P_{r}\left(p_{2}(\omega)\right.$ is attracting $)=P_{r}\left(\xi(\omega)>a-\frac{\mu}{\mu+(1-a)}\right)$.

4) If $\mu>a$ and $\mu>1-a$ then for any random fixed (periodic) point $p(\omega), P_{r}(p(\omega)$ is attracting) $=0$, and the system become noisy chaotic.

Proof: The proofs of (1), (2) and (3) are clear.

To prove (4), since $\left|\frac{\partial \tilde{T}_{a, \mu}\left(x_{t}, \xi_{t}(\omega)\right)}{\partial x_{t}}\right|= \begin{cases}\frac{\mu}{a} & \text { if } x_{t}<a \\ \frac{\mu}{1-a} & \text { if } x_{t}>a\end{cases}$

Then by lemma 1 any random fixed (periodic) point $p(\omega)$ is attracter with probability 0 .

To show that the system is noisy chaotic we will calculate stochastic Lyapunov exponent (SLE) as follows.

$\lambda_{s}\left(x_{0}\right)=\lim _{n \rightarrow \infty} \frac{1}{n} \sum_{j=0}^{n-1} \log \left|\frac{\partial \widetilde{T}_{a, \mu}\left(x_{j}, \xi_{j}(\omega)\right)}{\partial x_{0}}\right|$

And since $\left|\frac{\partial \tilde{T}_{a, \mu}\left(x_{j}, \xi_{j}(\omega)\right)}{\partial x_{0}}\right|>1$ then $\lambda_{s}\left(x_{0}\right)>0$ and the system is noisy chaotic.

Example 3.1: Let $\xi_{t}(\omega) \sim \operatorname{Gauss}(0,0.0025)$, for system (4)

Case 1: let $\mu=0.5, a=0.6$ then by theorem (3.1)-part 1, the system has one random fixed point $p(\omega) \sim \operatorname{Gauss}(0,0.0025)$ and $P_{r}(p(\omega)$ is attracting $)=P_{r}(\xi(\omega)<0.6)=P_{r}(z(\omega)<12) \cong 1$, where $z(\omega) \sim \operatorname{Gauss}(0,1)$, and the SLE of the system is about $\lambda_{s}=-0.1822$.

Case 2: let $\mu=a=0.7$ then by theorem (3.1)-part 2 , then the system has infinity number of random fixed points $p(\omega) \sim \operatorname{Gauss}\left(p^{*}, 0.0025\right)$, for all points $p^{*} \in[0, a]$, and the SLE of the system is about $\lambda_{s}=-4.0547 \times 10^{-5}$.

Case 3: let $\mu=0.5, a=0.4$ then by theorem (3.1)-part 3, the system has two random fixed points $p_{1}(\omega) \sim \operatorname{Gauss}(0,0.0025), p_{2} \sim \operatorname{Gauss}(0.4545,0.0025), \quad$ and $P_{r}\left(p_{1}(\omega)\right.$ is attracting $)=P_{r}(\xi(\omega)>0.4)=$ $P_{r}(z(\omega)>8) \cong 0, P_{r}\left(p_{2}(\omega)\right.$ is attracting $)=P_{r}(\xi(\omega)>-0.0545)=P_{r}(z(\omega)>-1.09)=0.86214$, and the SLE of the system is about $\lambda_{s}=-0.1822$.

Case 4: let $=0.7, a=0.4$, then by theorem (3.1)-part 4 , all fixed or periodic points in the system are replying with probability 1 , and the SLE of the system is about $\lambda_{s}=0.2691$.

Not that Stochastic Lyapunov exponent of the system (4) and deterministic Lyapunov exponent of the system (1) are the same, because of the partial derivative of system (4) is not dependent on $x_{t}(\omega)$. The satisfaction of the above properties can be seen from the bifurcation diagrams figure (7) .

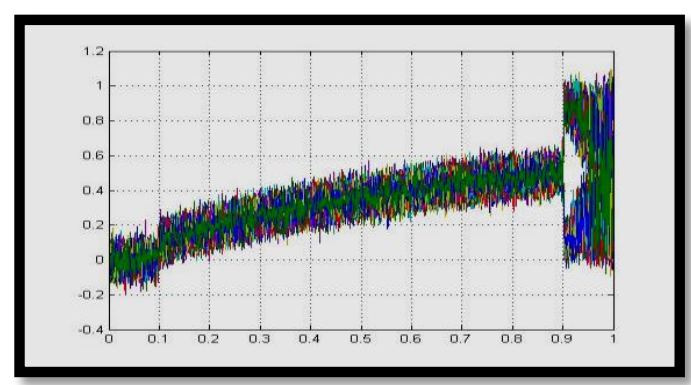

(a)

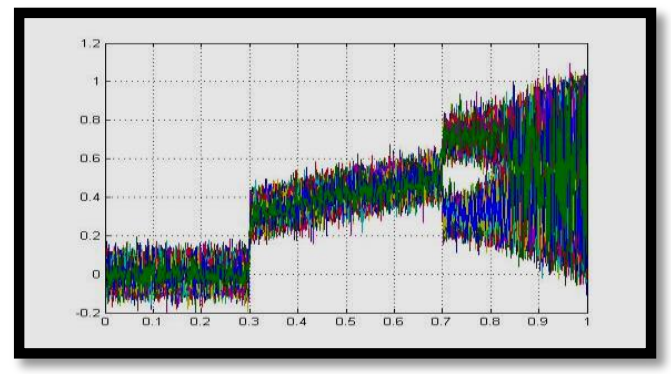

(c)

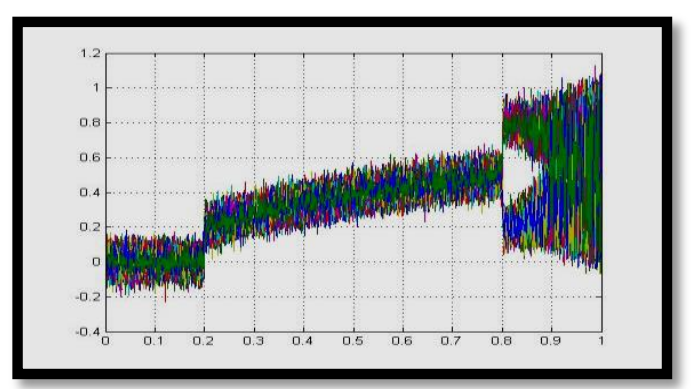

(b)

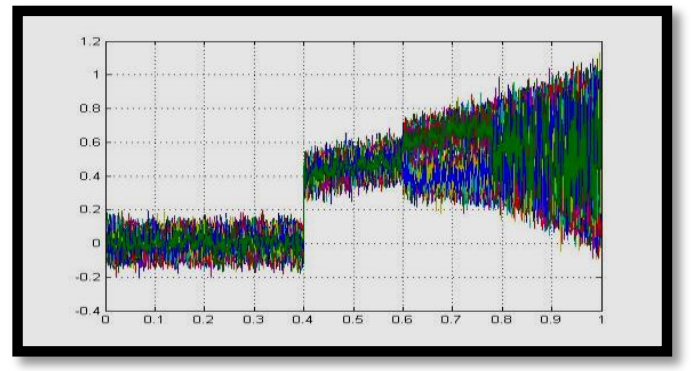

(d) 


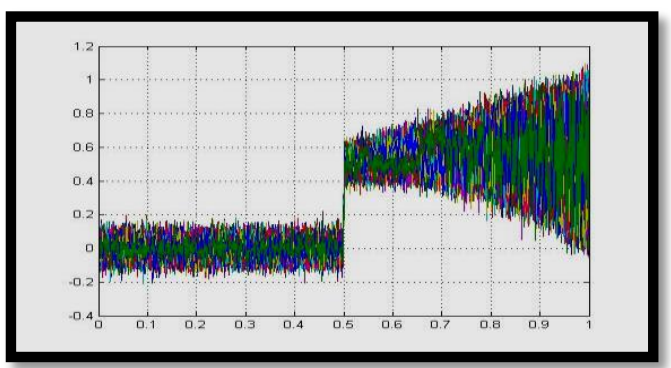

(e)

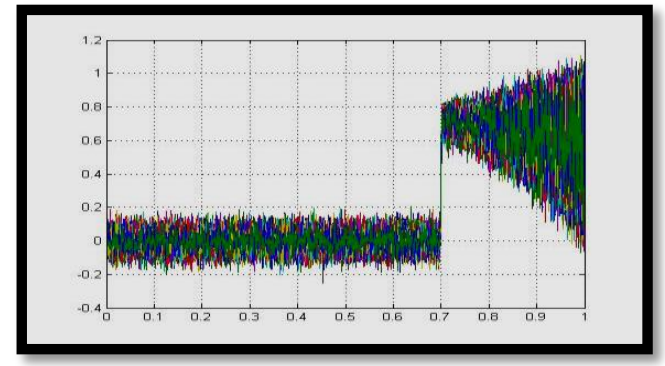

(g)

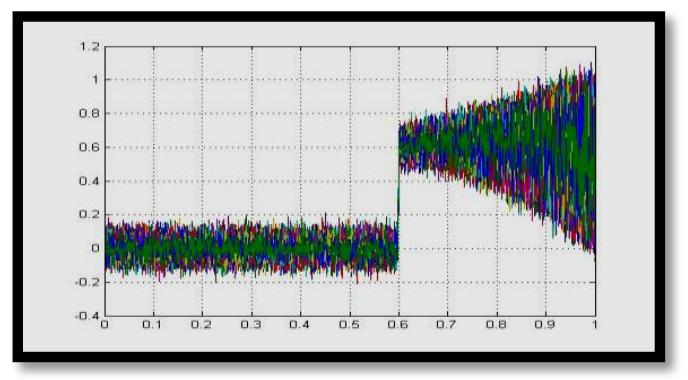

(f)

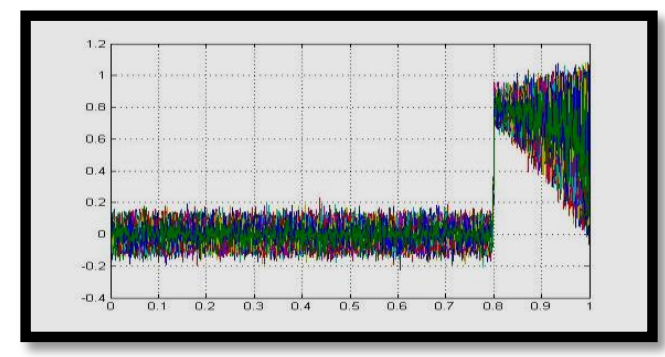

(h)

Fig. 7: bifurcation diagram of noisy asymmetric tent map family with additive noise Gauss $(0,0.0025)$, x-axes represent the values of $\mu$ and y-axes represent the values of $\mathrm{x}_{\mathrm{t}+1}\left(\omega_{)}\right.$, (a) $a=0.1$, (b) $a=0.2$, (c) $a=0.3$,(d) $a=0.4$, (e) $a=0.5$, (f) $a=0.6$, (g) $a=0.7$, (h) $a=0.8$.

\subsection{The family of noisy asymmetric logistic map.}

The noisy dynamical system for asymmetric logistic map with additive noise is given by.

$x_{t+1}(\omega)=\tilde{G}_{a, \mu}\left(x_{t}, \xi_{t}(\omega)\right)=\left\{\begin{array}{cc}\mu \frac{x_{t}}{2 a}\left(1-\frac{x_{t}}{2 a}\right)+\xi_{t}(\omega) & \text { if } 0 \leq x_{t} \leq a \\ \mu\left(\frac{x_{t}+1-2 a}{2-2 a}\right)\left(1-\left(\frac{x_{t}+1-2 a}{2-2 a}\right)\right)+\xi_{t}(\omega) & \text { if } a<x_{t} \leq 1\end{array}\right.$

Where $\tilde{G}_{a, \mu}: \mathbb{R} \times \Omega \rightarrow \mathbb{R},\left\{\xi_{t}(\omega)\right\}$ is a sequence of iid random variables and $0 \leq a \leq 1,0 \leq \mu \leq 4$.

Theorem 3.2: For the dynamical system (5), where $\xi_{t}(\omega) \sim \operatorname{Gauss}\left(0, \sigma^{2}\right)$.

1) If $\mu<2 a$, then the system has one random fixed point $p_{1}(\omega) \sim \operatorname{Gauss}\left(0, \sigma^{2}\right)$, and $P_{r}\left(p_{1}(\omega)\right.$ is attracting) $=$ $P_{r}(\xi(\omega)<a) * P_{r}\left(a-\frac{2 a^{2}}{\mu}<p_{1}(\omega)<a+\frac{2 a^{2}}{\mu}\right)+P_{r}(\xi(\omega)>a) * P_{r}\left(a-\frac{(2-2 a)^{2}}{\mu}<p_{1}(\omega)<a+\frac{(2-2 a)^{2}}{\mu}\right)$.

2) If $2 a<\mu<4 a$ then the system has another random fixed point $p_{2}(\omega) \sim \operatorname{Gauss}\left(\frac{2 a \mu-4 a^{2}}{\mu}, \sigma^{2}\right)$, where $\quad P_{r}\left(p_{2}(\omega)\right.$ is attracting $)=P_{r}\left(\xi(\omega)<a-\frac{2 a \mu-4 a^{2}}{\mu}\right) * P_{r}\left(a-\frac{2 a^{2}}{\mu}<p_{2}(\omega)<a+\frac{2 a^{2}}{\mu}\right)+P_{r}(\xi(\omega)>$ $\left.a-\frac{2 a \mu-4 a^{2}}{\mu}\right) * P_{r}\left(a-\frac{(2-2 a)^{2}}{\mu}<p_{2}(\omega)<a+\frac{(2-2 a)^{2}}{\mu}\right)$.

3) If $4 a<\mu$ then the second random fixed point

$p_{2}(\omega) \sim \operatorname{Gauss}\left(\frac{\left(\frac{2 a \mu}{(2-2 a)^{2}}-1\right)-\sqrt{\left(\frac{2 a \mu}{(2-2 a)^{2}}-1\right)^{2}+\left(\frac{4\left(\mu^{2}-2 a \mu^{2}\right)}{(2-2 a)^{4}}\right)}}{\frac{2 \mu}{(2-2 a)^{2}}}, \sigma^{2}\right)$

Is attracting with probability

$P_{r}\left(p_{2}(\omega)\right.$ is attracting $)=P_{r}\left(\xi(\omega)<a-\frac{\left(\frac{2 a \mu}{(2-2 a)^{2}}-1\right)-\sqrt{\left(\frac{2 a \mu}{(2-2 a)^{2}}-1\right)^{2}+\left(\frac{4\left(\mu^{2}-2 a \mu^{2}\right)}{(2-2 a)^{4}}\right)}}{\frac{2 \mu}{(2-2 a)^{2}}}\right) * P_{r}\left(a-\frac{2 a^{2}}{\mu}<p_{2}(\omega)<a+\right.$ $\left.\frac{2 a^{2}}{\mu}\right)+P_{r}\left(\xi(\omega)>a-\frac{\left(\frac{2 a \mu}{(2-2 a)^{2}}-1\right)-\sqrt{\left(\frac{2 a \mu}{(2-2 a)^{2}}-1\right)^{2}+\left(\frac{4\left(\mu^{2}-2 a \mu^{2}\right)}{(2-2 a)^{4}}\right)}}{\frac{2 \mu}{(2-2 a)^{2}}}\right) * P_{r}\left(a-\frac{(2-2 a)^{2}}{\mu}<p_{2}(\omega)<a+\frac{(2-2 a)^{2}}{\mu}\right)$.

Proof: Clear, by definition 1.2 and lemma 1.1.

Example 3.2: Let $\xi_{t}(\omega) \sim \operatorname{Gauss}(0,0.0025)$, for system (4) 
Case 1: let $=1, a=0.6$, by theorem (3.2)-part 1 , the system has one random fixed point $p_{1}(\omega) \sim \operatorname{Gauss}(0,0.0025)$, and it is attracting with probability $P_{r}(p(\omega)$ is attracting $)=P_{r}(\xi(\omega)<0.6) * P_{r}\left(-0.12<p_{1}(\omega)<1.32\right)+$ $P_{r}(\xi(\omega)>0.6) * P_{r}\left(-0.04<p_{1}(\omega)<1.24\right)=P_{r}(z(\omega)<12) * P_{r}(-2.4<z(\omega)<26.4)+P_{r}(z(\omega)>12) *$ $P_{r}(-0.8<z(\omega)<24.8) \cong 0.9918$, where $z(\omega) \sim \operatorname{Gauss}(0,1)$, and the SLE of system is about $\lambda_{s}=-0.1863$.

Case 2 : let $\mu=2, a=0.6$ by theorem (3.2)-part 2 , the system has two random fixed point $p_{1}(\omega) \sim \operatorname{Gauss}(0,0.0025)$ , $p_{2}(\omega) \sim \operatorname{Gauss}(0.48,0.0025)$, and $P_{r}\left(p_{1}(\omega)\right.$ is attracting $)=P_{r}(\xi(\omega)<0.6) * P_{r}\left(0.24<p_{1}(\omega)<0.96\right)+$ $P_{r}(\xi(\omega)>0.6) * P_{r}\left(0.28<p_{1}(\omega)<0.92\right)=P_{r}(z(\omega)<12) * P_{r}(4.8<z(\omega)<19.2)+P_{r}(\xi(\omega)>12) *$ $P_{r}(5.6<z(\omega)<18.4) \cong 0 \quad, \quad P_{r}\left(p_{2}(\omega)\right.$ is attracting $)=P_{r}(\xi(\omega)<0.12) * P_{r}\left(0.24<p_{2}(\omega)<0.96\right)+$ $P_{r}(\xi(\omega)>0.12) * P_{r}\left(0.28<p_{2}(\omega)<0.92\right)=P_{r}(z(\omega)<2.4) * P_{r}(-4.8<z(\omega)<9.6)+P_{r}(z(\omega)>2.4) *$ $P_{r}\left(-4<p_{2}(\omega)<8.8\right) \cong 1$, where $z(\omega) \sim \operatorname{Gauss}(0,1)$ and the SLE of the system is about $\lambda_{s}=-1.2356$. Case 3: let $=2.5, a=0.6$, by theorem (3.2)-part 3, the second fixed point $p_{2}(\omega) \sim \operatorname{Gauss}(0.624,0.0025)$, and $P_{r}\left(p_{2}(\omega)\right.$ is attracting $)=P_{r}(\xi(\omega)<-0.024) * P_{r}\left(0.312<p_{2}(\omega)<0.888\right)+P_{r}(\xi(\omega)>-0.024) *$ $P_{r}\left(0.344<p_{2}(\omega)<0.856\right)=P_{r}(z(\omega)<-0.48) * P_{r}(-6.24<z(\omega)<5.28)+P_{r}(z(\omega)>-0.48) * P_{r}(-5.6<$ $z(\omega)<4.64) \cong 1$, where $z(\omega) \sim \operatorname{Gauss}(0,1)$, and the SLE of the system is about $\lambda_{s}=-1.7190$. In this example the approximate value of $\mu_{n c}=3.523$.

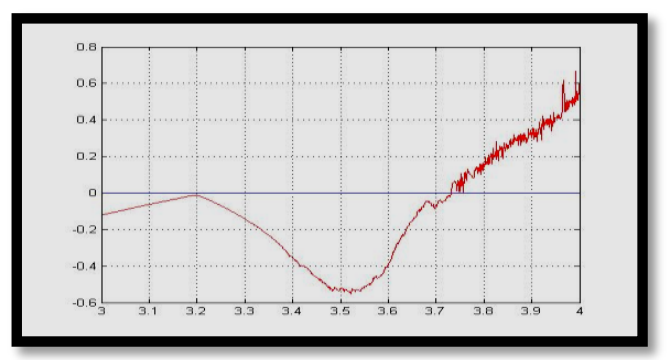

(a)

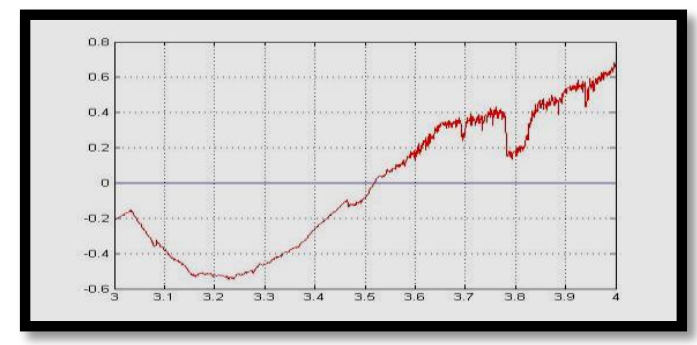

(c)

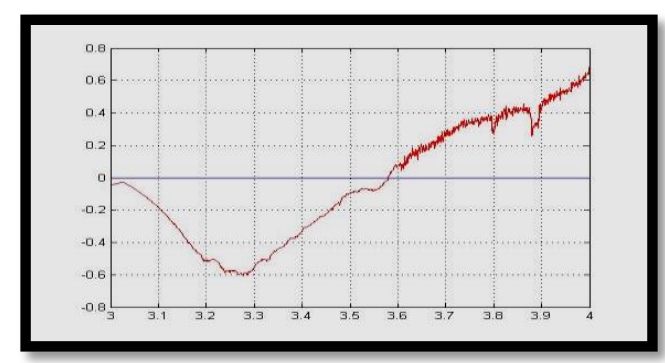

(b)

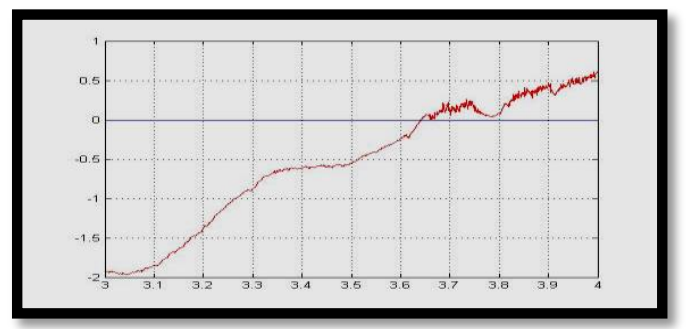

(d)

Fig. 8: S.L.E. for noisy asymmetric logistic map with additive Gauss $(0,0.0025)$ noise, $x_{-}$axes represent the values of $\mu$ and $y_{-}$axes represent the values of S.L.E., (a) $a=2$, (b) $a=0.4$, (c) $a=0.6$, (d) $a=0.8$.

The satisfaction of the above properties can be seen from the bifurcation diagrams figure (9).

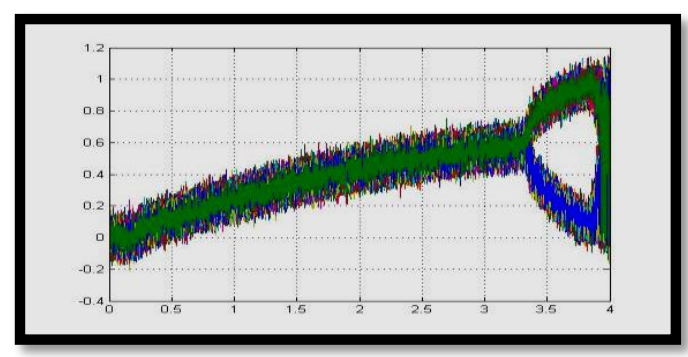

(a)

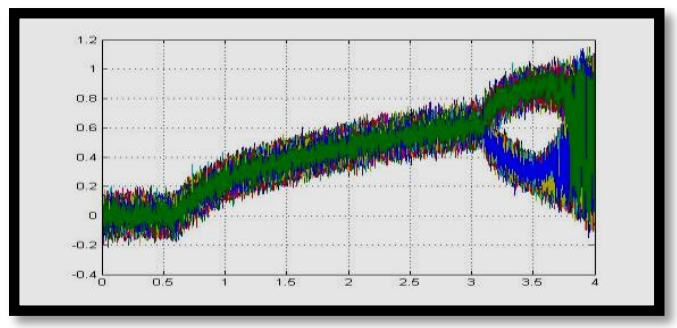

(c)

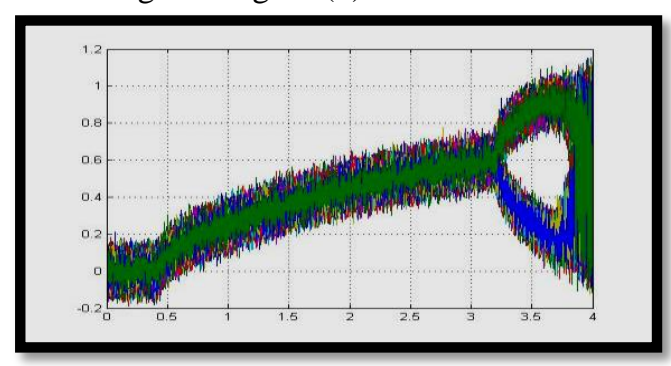

(b)

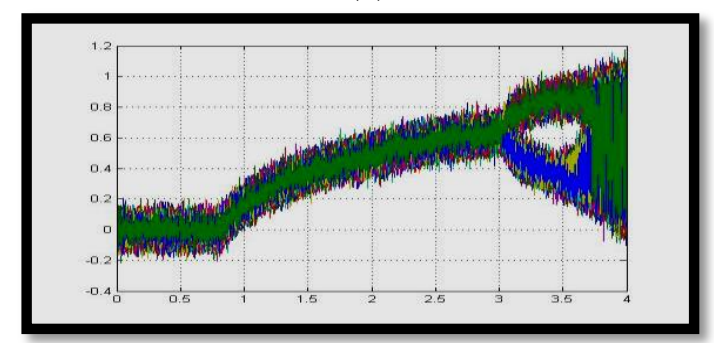

(d) 


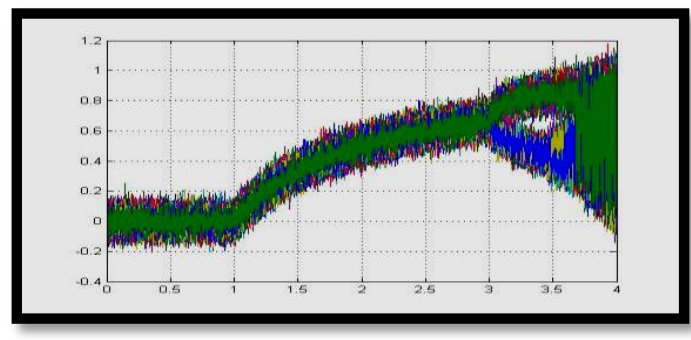

(e)

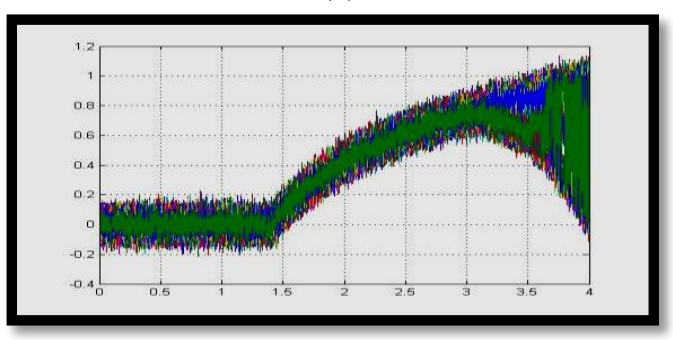

(g)

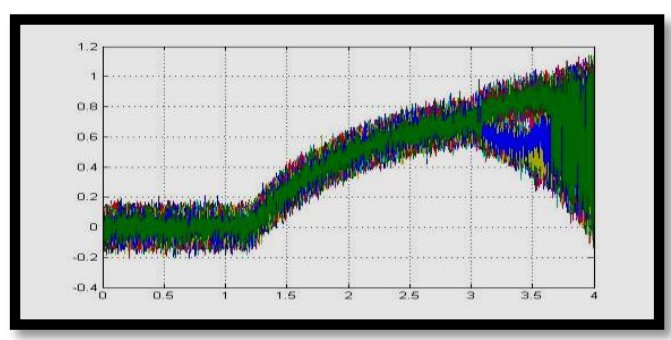

(f)

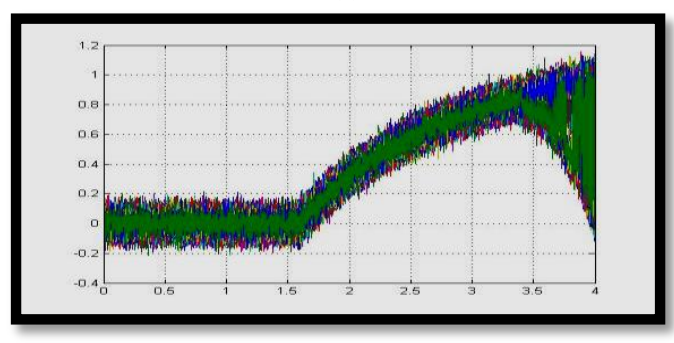

(h)

Fig. 9: bifurcation diagram of noisy asymmetric logistic map family with additive noise Gauss $(0,0.0025)$, x-axes represent the values of $\mu$ and $y$ axes represent the values of $\mathrm{x}_{\mathrm{t}+1}(\omega)$ (a) $a=0.1$, (b) $a=0.2$, (c) $a=0.3$,(d) $a=0.4$, (e) $a=0.5$, (f) $a=0.6$,(g) $a=0.7$, (h) $a=0.8$

\subsection{The family of noisy mixed logistic-tent map.}

The noisy dynamical system for mixed logistic-tent map with additive noise is given by:

$x_{t+1}(\omega)=\widetilde{G T}_{a, \mu}\left(x_{t}, \xi_{t}(\omega)\right)=\left\{\begin{array}{cc}\mu \frac{x_{t}}{2 a}\left(1-\frac{x_{t}}{2 a}\right)+\xi_{t}(\omega) & \text { if } 0 \leq x_{t} \leq a \\ \mu\left(\frac{1-x_{t}}{4(1-a)}\right)+\xi_{t}(\omega) & \text { if } a<x_{t} \leq 1\end{array}\right.$

Where $\widetilde{G T}_{a, \mu}: \mathbb{R} \times \Omega \rightarrow \mathbb{R},\left\{\xi_{t}(\omega)\right\}$ sequence of (iid) random variables and $0 \leq a \leq 1,0 \leq \mu \leq 4$

Theorem 3.3: For the dynamical system (6), if $\xi_{t}(\omega) \sim \operatorname{Gauss}\left(0, \sigma^{2}\right)$.

1) If $\mu<2 a$, then the system has one random fixed point $p_{1}(\omega) \sim \operatorname{Gauss}\left(0, \sigma^{2}\right)$, and $P_{r}\left(p_{1}(\omega)\right.$ is attracting $)=$ $P_{r}(\xi(\omega)<a) * P_{r}\left(a-\frac{2 a^{2}}{\mu}<p_{1}(\omega)<a+\frac{2 a^{2}}{\mu}\right)+P_{r}(\xi(\omega)>a)$, if $<4(1-a)$, and if $\mu>4(1-a)$ then $P_{r}\left(p_{1}(\omega)\right.$ is attracting $)=P_{r}(\xi(\omega)<a) * P_{r}\left(a-\frac{2 a^{2}}{\mu}<p_{1}(\omega)<a+\frac{2 a^{2}}{\mu}\right)$.

2) If $2 a<\mu \leq 4 a$ then the system has another random fixed points $p_{2}(\omega) \sim \operatorname{Gauss}\left(\frac{2 a \mu-4 a^{2}}{\mu}, \sigma^{2}\right)$, and $P_{r}\left(p_{2}(\omega)\right.$ is attracting $)=P_{r}\left(\xi(\omega)<a-\frac{2 a \mu-4 a^{2}}{\mu}\right) * P_{r}\left(a-\frac{2 a^{2}}{\mu}<p_{2}(\omega)<a+\frac{2 a^{2}}{\mu}\right)+P_{r}(\xi(\omega)>a-$

$\left.\frac{2 a \mu-4 a^{2}}{\mu}\right)$, if $\mu<4(1-a)$ and if $\mu>4(1-a)$, then $P_{r}\left(p_{2}(\omega)\right.$ is attracting $)=P_{r}\left(\xi(\omega)<a-\frac{2 a \mu-4 a^{2}}{\mu}\right) *$ $P_{r}\left(a-\frac{2 a^{2}}{\mu}<p_{2}(\omega)<a+\frac{2 a^{2}}{\mu}\right)$.

$3)$ If $4 a<\mu<4(1-a)$ then the second random fixed point $p_{2}(\omega) \sim \operatorname{Gauss}\left(\frac{\mu}{\mu+4(1-a)}, \sigma^{2}\right)$ is attracting with probability $\quad P_{r}\left(p_{2}(\omega)\right.$ is attracting $)=P_{r}\left(\xi(\omega)<a-\frac{\mu}{\mu+4(1-a)}\right) * P_{r}\left(a-\frac{2 a^{2}}{\mu}<p_{2}(\omega)<a+\frac{2 a^{2}}{\mu}\right)+$ $P_{r}\left(\xi(\omega)>a-\frac{\mu}{\mu+4(1-a)}\right)$, and $P_{r}\left(p_{2}(\omega)\right.$ is attracting $)=P_{r}\left(\xi(\omega)<a-\frac{\mu}{\mu+4(1-a)}\right) * P_{r}\left(a-\frac{2 a^{2}}{\mu}<p_{2}(\omega)<\right.$ $\left.a+\frac{2 a^{2}}{\mu}\right)$ if $\mu>\max \{4 a, 4(1-a)\}$.

Proof: The proofs of (1) and (3) is in the similar way of (2)

To prove (2), let $p_{2}(\omega)=\widetilde{G T}_{a, \mu}(p, \xi(\omega))=\mu \frac{p}{2 a}\left(1-\frac{p}{2 a}\right)+\xi(\omega)=\frac{2 a \mu-4 a^{2}}{\mu}+\xi(\omega)$, since $2 a<\mu \leq 4 a \operatorname{Implies} p_{2}(\omega) \sim$ Gauss $\left(\frac{2 a \mu-4 a^{2}}{\mu}, \sigma^{2}\right)$.

Now to find probability of $p_{2}(\omega)$ is attracter

$P_{r}\left(p_{2}(\omega)\right.$ is attracting $)=P_{r}\left(p_{2}(\omega)<a\right) * P_{r}\left(p_{2}(\omega)\right.$ is attracting $\left.\mid p_{2}(\omega)<a\right)+P_{r}\left(p_{2}(\omega)>a\right) *$ $P_{r}\left(p_{2}(\omega)\right.$ is attracting $\left.\mid p_{2}(\omega)>a\right)$

We know that if $\mu<4(1-a)$ then $P_{r}\left(p_{2}(\omega)\right.$ is attracting $\left.\mid p_{2}(\omega)>a\right)=1$

Then if $\mu<4(1-a)$ 
$P_{r}\left(p_{2}(\omega)\right.$ is attracting $)=P_{r}\left(\xi(\omega)<a-\frac{2 a \mu-4 a^{2}}{\mu}\right) * P_{r}\left(a-\frac{2 a^{2}}{\mu}<p_{2}(\omega)<a+\frac{2 a^{2}}{\mu}\right)+P_{r}(\xi(\omega)>\quad a-$ $\left.\frac{2 a \mu-4 a^{2}}{\mu}\right)$, by lemma 1 and if $\mu>4(1-a) P_{r}\left(p_{2}(\omega)\right.$ is attracting $\left.\mid p_{2}(\omega)>a\right)=0$ and hence $P_{r}\left(p_{2}(\omega)\right.$ is attracting $)=P_{r}\left(\xi(\omega)<a-\frac{2 a \mu-4 a^{2}}{\mu}\right) * P_{r}\left(a-\frac{2 a^{2}}{\mu}<p_{2}(\omega)<a+\frac{2 a^{2}}{\mu}\right)$

Example 3.3: Let $\xi_{t}(\omega) \sim \operatorname{Gauss}(0,0.0025)$, for system (6)

Case 1: let $=1, a=0.6$, by theorem (3.3)-part 1, the system has on random fixed point $p(\omega) \sim \operatorname{Gauss}(0,0.0025)$, and it is attracting with probability $P_{r}(p(\omega)$ is attracting $)=P_{r}(\xi(\omega)<0.6) * P_{r}\left(-0.12<p_{1}(\omega)<1.32\right)+$ $P_{r}(\xi(\omega)>a)=P_{r}(z(\omega)<12) * P_{r}(-2.4<z(\omega)<26.4)+P_{r}(z(\omega)>12) \cong 0.9918$, where $z(\omega) \sim \operatorname{Gauss}(0,1)$ and the SLE of system is about $\lambda_{s}=-0.1869$.

Case 2: let $\mu=2, a=0.6$ by theorem (3.3)-part 2, the system has another random fixed point $p_{2}(\omega) \sim$ Gauss $(0.48,0.0025), \quad$ and $P_{r}\left(p_{2}(\omega)\right.$ is attracting $)=P_{r}(\xi(\omega)<0.12) * P_{r}\left(0.24<p_{2}(\omega)<0.96\right)=$ $P_{r}(z(\omega)<2.4) * P_{r}(-4.8<z(\omega)<9.6) \cong 0.9918$, where $z(\omega) \sim \operatorname{Gauss}(0,1)$ and the SLE of the system is about $\lambda_{s}=-1.2148$.

Case 3: let $=2.5, a=0.6$, by theorem (3.3)-part 3 in above the second random fixed point $p_{2}(\omega) \sim$ Gauss $(0.6098,0.0025) \quad, \quad$ and $P_{r}\left(p_{2}(\omega)\right.$ is attracting $)=P_{r}(\xi(\omega)<-0.0098) * P_{r}\left(0.312<p_{2}(\omega)<\right.$ $0.888)=P_{r}(z(\omega)<-0.196) * P_{r}(-5.956<z(\omega)<5.564)=0.422695$, and the SLE of the system is about $\lambda_{s}=-0.9515$.

In this example the approximate value of $\mu_{n c}=3.1836$.

The satisfaction of the above properties can be seen from the bifurcation diagrams figure (11).

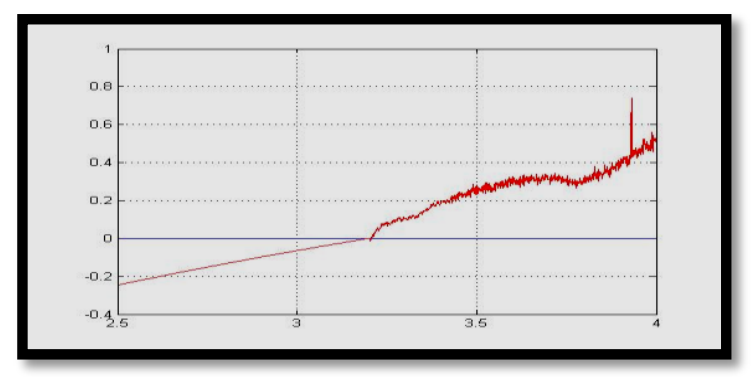

(a)

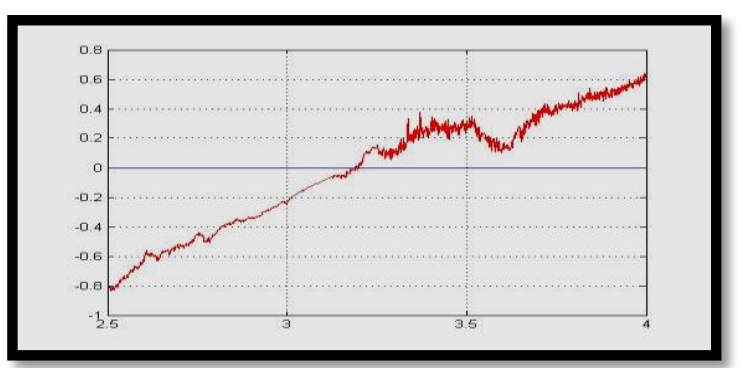

(c)

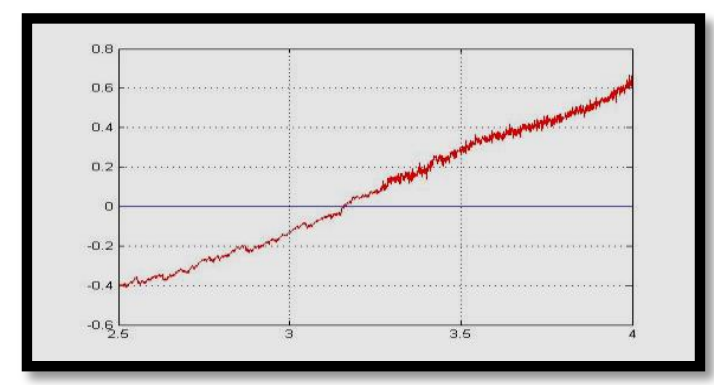

(b)

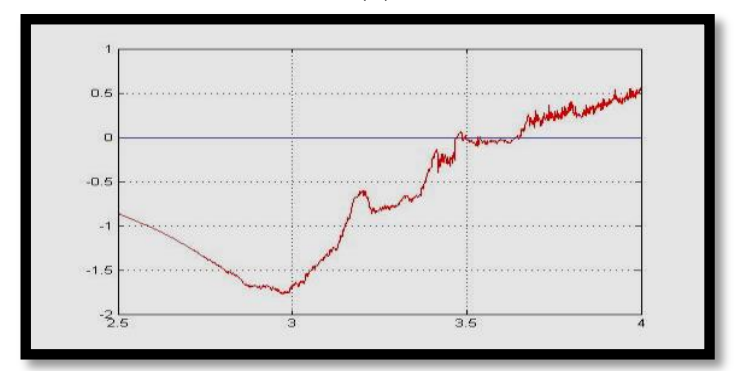

(d)

Fig. 10: SLE for noisy mixed logistic-tent map with additive Gauss $(0,0.0025)$ noise , $x_{-}$axes represent the values of value of $\mu$ and $y_{-}$axes represent the values value of S.L.E. , (a) $a=2$, (b) $a=0.4$, (c) $a=0.6$, (d) $a=0.8$.

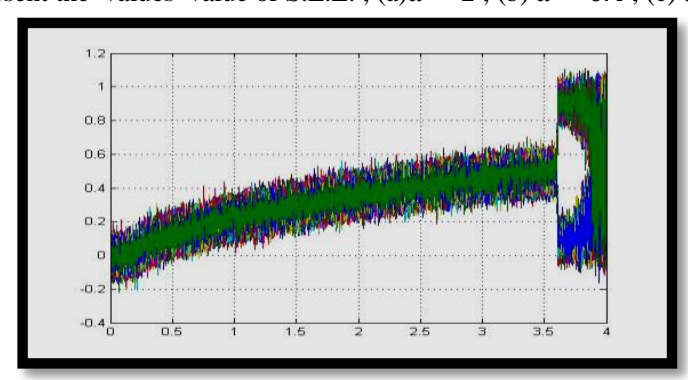

(a)

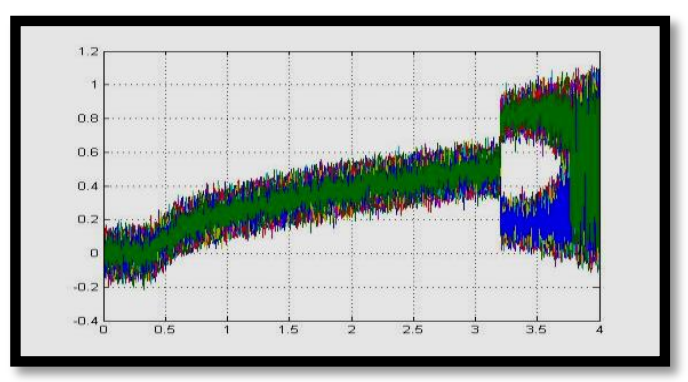

(b) 


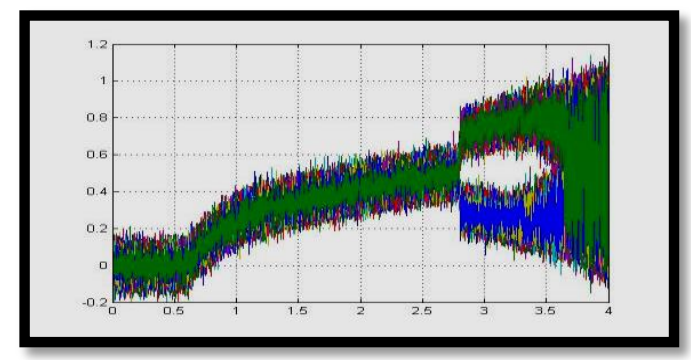

(c)

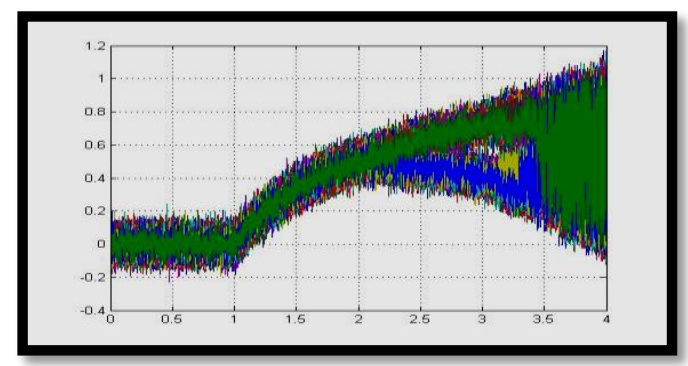

(e)

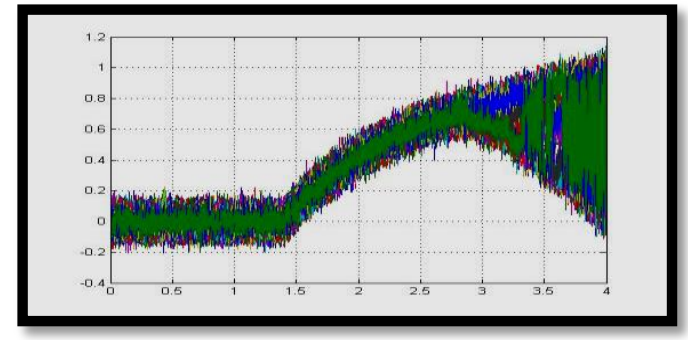

(g)

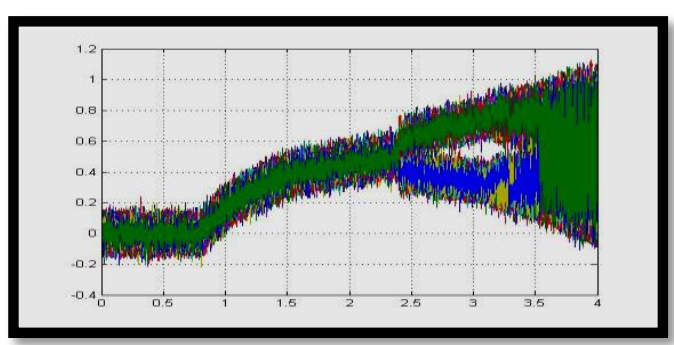

(d)

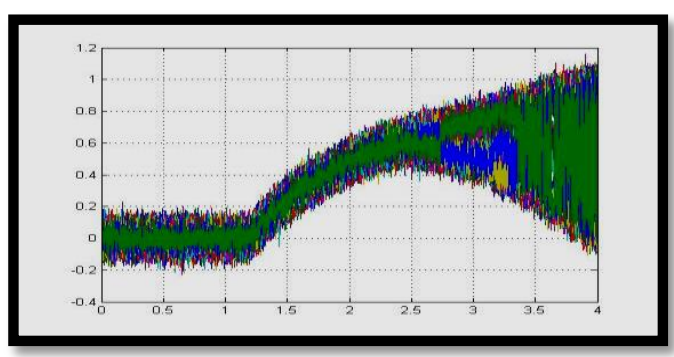

(f)

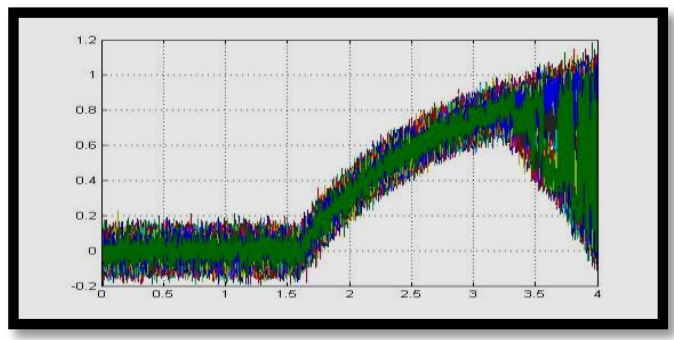

(h)

Fig. 11: bifurcation diagram of noisy mixed logistic-tent map family with additive noise Gauss $(0,0.0025)$, x-axes represent the values of $\mu$ and $y$ axes represent the values of $\mathrm{x}_{\mathrm{t}+1}(\omega)$, (a) $a=0.1$, (b) $a=0.2$, (c) $a=0.3$, (d) $a=0.4$, (e) $a=0.5$, (f) $a=0.6$, (g) $a=0.7$, (h) $a=0.8$.

\section{References}

[1] Arnold, L. Random dynamical system. Springer-Varlag, Berlin, (1998).

[2] Chan, K. and Tong, T.Chaos: a statistical perspective. Springer-Varlag, New York, (2001).

[3] Ferrari, F. and Souza pinto, S. The skew tent map entropy production. Dynamics day's South America. July (2010).

[4] Ichimura, K. And Ito, M. Dynamics of skew tent maps. RIMS Kokyuroku, (1998)1042:92-98.

[5] Lindstrom, T. and Thunberg, H. . An elementary approach to dynamics and bifurcations of skew tent maps . Journal of difference equations and applications (2008) 14. 819-833.

[6] Schenk-Hoppé, K. R. Sample-path stability of non-stationary dynamic economic systems. Institute for empirical research in economics, University of Zurich, Working paper series ISSN 1424-0459, working paper No. 46. March (2001). 